А. Умба' ${ }^{1}$, магистр , Г. В. Сарибога ${ }^{2}$, старший преподаватель, О. В. Збруцький ${ }^{3}$, професор, д.т.н.

\title{
СИНТЕЗ АЛГОРИТМА ПОВЫШЕНИЯ НАДЕЖНОСТИ РАСПОЗНАВАНИЯ ОРИЕНТИРА СИСТЕМОЙ ТЕХНИЧЕСКОГО ЗРЕНИЯ
}

En The development of the method for correction of errors in the inertial navigation system (INS) by means and methods of technical vision (MTV) is an actual and effective application problem.

Having the values of the distance and direction of the UAV to the benchmark, the UAV control algorithm is being developed to minimize the difference between the centers of the object and the optical axis of the camera. The MTV after each image processing cycle gives new values of the distance and direction of the UAV to the reference point, which corrects the movement of the UAV to it.

The performed tests of the working capacity of the developed algorithm on the mobile platform showed an increase in the accuracy of the INS by 1,8 times.

Розробка методу корекції помилок інерціальної навігаційної системи (IHC) засобами та методами технічного зору є актуальною та ефективною прикладною задачею.

Імея значення дистанції та напрямку БПЛА до орієнтиру, обробляється алгоритм управління БПЛА для мінімізації розбіжностей між центрами об'єкту та оптичною осі камери. СТЗ після кожного циклу обробки зображення видає нові значення дистанцій та напрямку БПЛА до орієнтиру, які коригують рух до неї БПЛА.

Проведені тестові випробування роботоспособності розробленого алгоритму на мобільній платформі показали підвищені точності IHC у 1,8 раза.

\footnotetext{
${ }^{1}$ Университет г. Льовин, Польша

${ }^{2}$ НТУУ «Київський політехнічний інститут ім. Ігоря Сікорського», кафедра приборов и систем управления летательными аппаратами

${ }^{3}$ НТУУ «Київький політехнічний інститут ім. Ігоря Сікорського», кафедра приборов и систем управления летательньми аппаратами
} 


\section{Введение}

Существующие методы распознавания ориентира системой технического зрения (СТ3) содержат много вариантов решений, из которых лишь некоторые являются оптимальными [1].

Применение алгоритмов, реализующих работу СТ3 [2] в значительной степени зависит от условий эксплуатации распознавания.

Анализ публикаций [2 - 8] показывает, что при разработке алгоритмов СТЗ часто не учитывается надежность распознавания ориентира. Статистические испытания разработанных алгоритмов $[2,3,5,7]$ показывают их неустойчивость к воздействию помех. Невозможность обеспечить требуемую величину надежности распознавания ориентира обусловила необходимость разработки алгоритма повышения надежности распознавания ориентира системой технического зрения.

\section{Постановка задачи}

Целью статьи является синтез алгоритма повышения надежности распознавания ориентира системой технического зрения при различных условиях эксплуатации и внешних воздействиях на ориентир.

\section{Синтез алгоритма распознавания ориентира}

Так как надежность распознавания ориентира применением только одного метода из известных недостаточна, синтезируем алгоритм распознавания ориентира комплексированием известных методов распознавания $[2,3]$ с применением фильтра Калмана. В качестве распознаваемого ориентира используем стандартный символ взлётно-посадочной площадки (символ Н в окружности).

Осуществим первичную обработку изображения методом выделения контура, используя моментные функции, $h u$-моменты $[3,9-10]$, инвариантные к смещению, масштабу и повороту ориентира, что дает возможность распознавания контура ориентира.

Но, поскольку при использовании данного метода могут быть совпадения по контуру с другими объектами, применяем также методы распознавания по особым точкам (рис. 1), которые определяются на символе $\mathrm{H:} \mathrm{методы} \mathrm{Лукаса-Канаде}[4,11]$ и преобразование Хафа $[4,11]$.

При вторичной обработке мы предотвращаем некорректное обнаружение ключевых точек при перемещении методом RANSAC [5], который заключается в том, что по имеющимся данным о точках строятся модели («гипотезы»). При этом все исходные данные делятся на два типа: удовлетворяющие модели, «не выбросы» («правильные точки») и ложные точки, искаженные, случайные включения в исходные данные, «выбросы». 
Данные алгоритмы обеспечивают на выходе траекторию как набор смещений точки между кадрами. Но при этом обладают медленной скоростью и некорректным обнаружением точек при переходе от одного кадра к другому. Изменение освещения или резкие движения камеры приводит в результате к искаженному распознаванию ориентира (рис. 2).

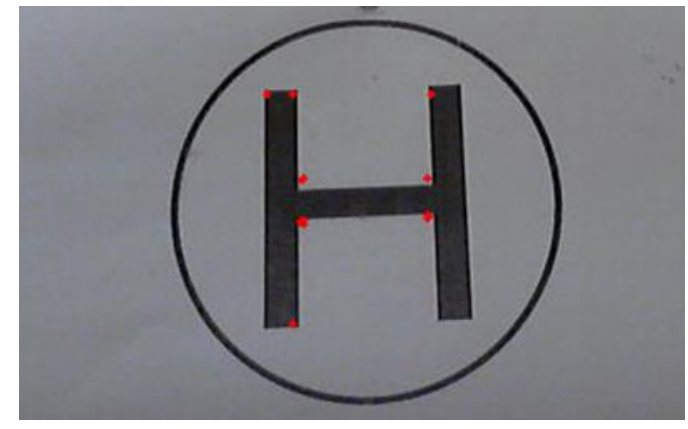

Рис. 1. Определение ключевых то- Рис. 2. Определение ключевых точек методом Лукаса-Канаде и преобразованием Хафа

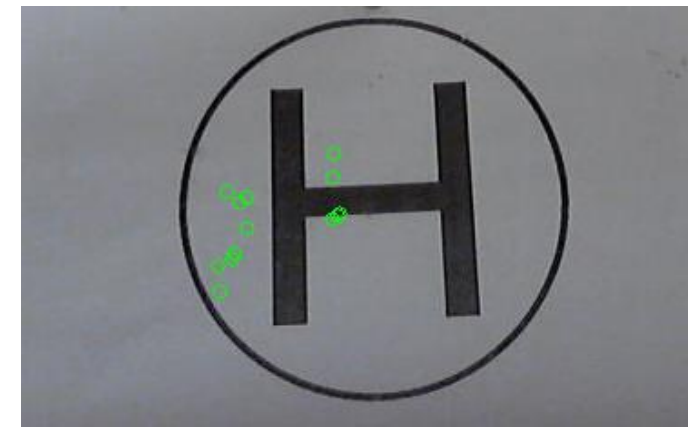
чек при переходе от одного кадра к другому

По результатам теста надежность распознавания не обеспечивается известными алгоритмами, поэтому возникает необходимость дополнительной фильтрации данных, которая осуществляется с помощью дискретного адаптивного фильтра Калмана второго порядка (ФК) [6].

Фильтр Калмана $[6,7]$ базируется на дискретных по времени линейных динамических системах. В нашем случае возможно применение ФК для вычисления положения ориентиров, так как они удовлетворяют свойству линейности. Реализуем ФК для определения местоположения каждой ключевой точки, ее скорость и ускорение обозначены соответственно: $x_{k}, y_{k}, \mathrm{v}_{x k}, \mathrm{v}_{y k}, a_{x k}, a_{y k}$, где индекс $k$ обозначает $k$-ю ключевую точку.

Под моделью процесса подразумеваем, что истинное состояние ключевой точки в момент $k$ получается из истинного состояния в момент $(k-1)$ в соответствии с уравнением:

$$
x_{k}=\Phi_{k} x_{k-1}^{+}+\mathrm{B}_{k} \mathrm{U}_{k}+w_{k},
$$

где $x_{k}$ - предсказание состояния системы в текущий момент времени,

$\Phi_{k}$ - матрица перехода между состояниями,

$x_{k-1}^{+}-$состояние системы в предыдущий момент времени,

В - матрица применения управляющего воздействия,

$\mathrm{U}$ - матрица управляющего воздействия в данный момент времени

$w_{k}$ - ковариация шума процесса.

В момент $k$ производим наблюдение (измерение) $z_{k}$ истинного вектора состояния $x_{k}$. Вектора связаны между собой уравнением: 


$$
z_{k}=\mathrm{H} x_{k}+\mathrm{v}_{k}
$$

где $z_{k}$ - измерения в текущий момент времени,

H - матрица измерений, отображающая отношения измерений и состояния,

$\mathrm{v}_{k}$ - матрица ковариации шума измерения.

Представляем ключевую точку в пространстве состояний:

$$
\left[\begin{array}{c}
x_{k} \\
y_{k} \\
\mathrm{v}_{x_{k}} \\
\mathrm{v}_{y_{k}} \\
a_{x_{k}} \\
a_{y_{k}}
\end{array}\right]=\left[\begin{array}{cccccc}
1 & 0 & \Delta t & 0 & \frac{1}{2} \Delta t^{2} & 0 \\
0 & 1 & 0 & \Delta t & 0 & \frac{1}{2} \Delta t^{2} \\
0 & 0 & 1 & 0 & \Delta t & 0 \\
0 & 0 & 0 & 1 & 0 & \Delta t \\
0 & 0 & 0 & 0 & 1 & 0 \\
0 & 0 & 0 & 0 & 0 & 1
\end{array}\right]\left[\begin{array}{c}
x_{k-1} \\
y_{k-1} \\
\mathrm{v}_{x_{k-1}} \\
\mathrm{v}_{y_{k-1}} \\
a_{x_{k-1}} \\
a_{y_{k-1}}
\end{array}\right]+\left[\begin{array}{c}
10 \\
10 \\
125 \\
125 \\
1562,5 \\
1562,5
\end{array}\right],
$$

где $x_{k}, y_{k}$ - координаты ключевой точки, $\mathrm{v}_{x k}, \mathrm{v}_{y k}$ - проекции вектора скорости ключевой точки, $a_{x k}, a_{y k}$ - проекции ускорения особой точки.

При использовании метода определения позиции ключевой точки уменьшается дисперсия гауссовского распределения, что приводит к уменьшению неопределенности при измерениях [4]. Ковариационная матрица шума измерений $R$ :

$$
R=\left[\begin{array}{ll}
1 & 0 \\
0 & 1
\end{array}\right]
$$

Ковариационная матрица шума процесса измерений $Q$ отображает насколько в нашем случае положение и скорость точки могут меняться вдоль оси $x$ и $y$ между двумя последующими измерениями. Средний дискретный шаг времени без оптимизации программного обеспечения составляет $t=0,08 \mathrm{c}$. Наибольшее расстояние, на которое ключевые точки могут перемещаться за это время, равно $r=10$ пикселям в пространстве изображения. Исходя из заданных параметров, определяем скорость перемещения и ускорение ключевой точки по оси х:

$$
\begin{gathered}
\mathrm{v}_{x_{k}}=\frac{r}{t}=\frac{10}{0,08}=125, \\
a_{x_{k}}=\frac{\mathrm{v}_{x_{k}}}{t}=\frac{125}{0,08}=1562,5 .
\end{gathered}
$$

Далее мы составляем ковариационную матрицу шума процесса измерений, где все недиагональные значения матрицы равны 0 , поскольку 
нет взаимосвязи между измерениями, а ненулевыми значениями являются только те, на которые влияет изменение положения ключевой точки по оси $x$ :

$$
Q=\left[\begin{array}{cccccc}
10 & 0 & 0 & 0 & 0 & 0 \\
0 & 10 & 0 & 0 & 0 & 0 \\
0 & 0 & 125 & 0 & 0 & 0 \\
0 & 0 & 0 & 125 & 0 & 0 \\
0 & 0 & 0 & 0 & 1562,5 & 0 \\
0 & 0 & 0 & 0 & 0 & 1562,5
\end{array}\right]
$$

Определяем текущее положение точки по оценке предыдущего ее состояния:

$$
\begin{gathered}
x_{k_{p}}=\Phi_{k} x_{k}+\mathrm{B}_{k} U_{k}+w_{k} ; \\
P_{k_{p}}=\Phi_{k} P_{k-1} \Phi_{k}^{T}+Q_{k} .
\end{gathered}
$$

Выполняем коррекцию прогнозируемого положения ключевой точки:

$$
\begin{gathered}
K_{k}=P_{k_{p}} \mathrm{H}^{T}\left(\mathrm{H} P_{k_{p}} \mathrm{H}^{T}+R_{k}\right)^{-1} \\
x_{k_{c}}=x_{k_{p}}+K_{k}\left[y-\mathrm{H} x_{k_{p}}\right] \\
P_{k_{c}}=\left(I-K_{k} \mathrm{H}\right) P_{k_{p}} .
\end{gathered}
$$

На рис. 3 отображено перемещение ключевой точки по координате х и результат применения ФК для этой точки при условии, что камера неподвижна.

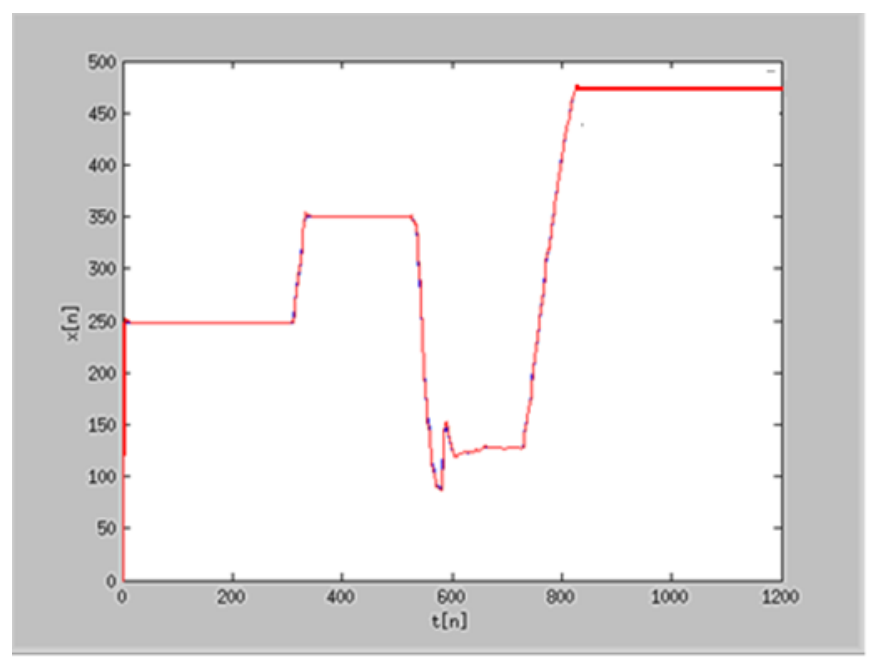

Рис. 3. Сигнал на выходе ФК

Наблюдается полное совпадение перемещения ключевой точки с применением ФК и работой известных алгоритмов. При возникновении 
16

препятствия, полностью закрывающего изображение, камера теряет ориентир. Это приводит к тому, что ключевые точки будут некорректно распознаны в следующих кадрах изображения при использовании известных алгоритмов (рис. 4). Однако, в нашем случае, благодаря ФК мы продолжаем определять и прогнозировать перемещение ключевой точки (рис. 4).

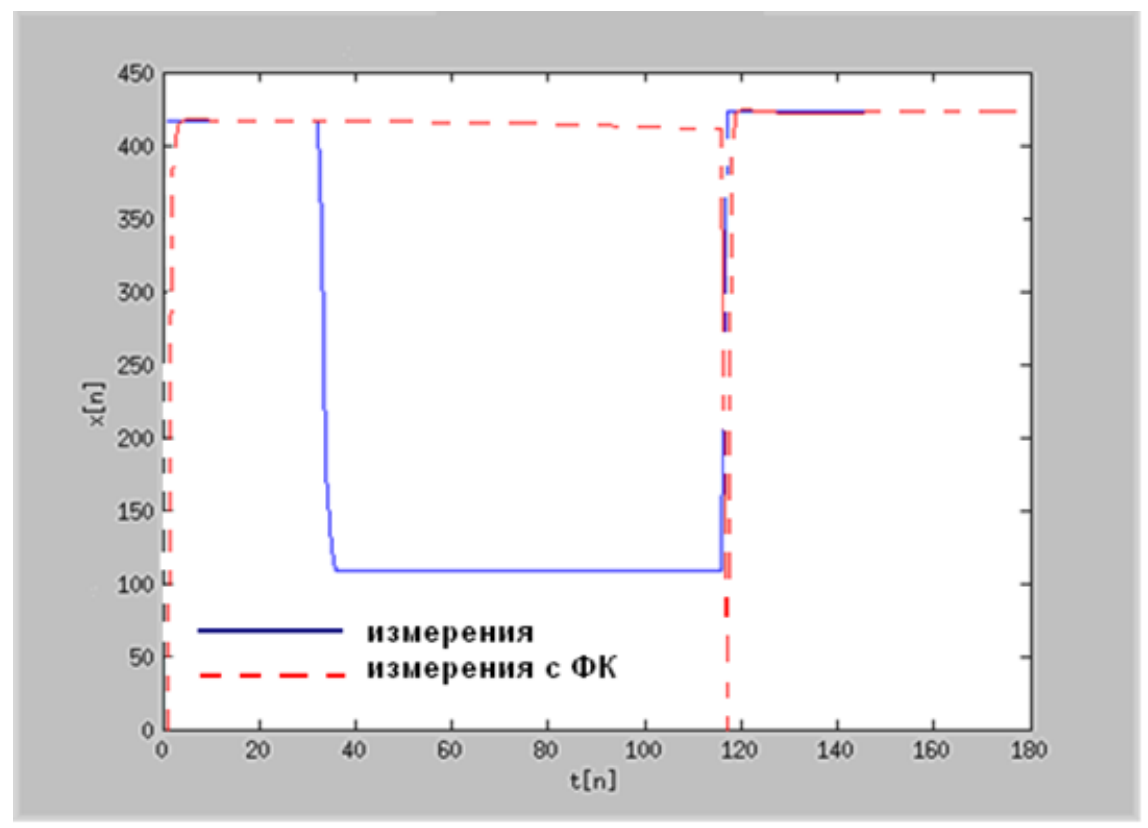

Рис. 4. Сигнал на выходе ФК с препятствием

При возникновении препятствия координаты ключевых точек пересчитываем (рис. 5). После проведения 3-х итераций успешно определяем все ключевые точки ориентира.

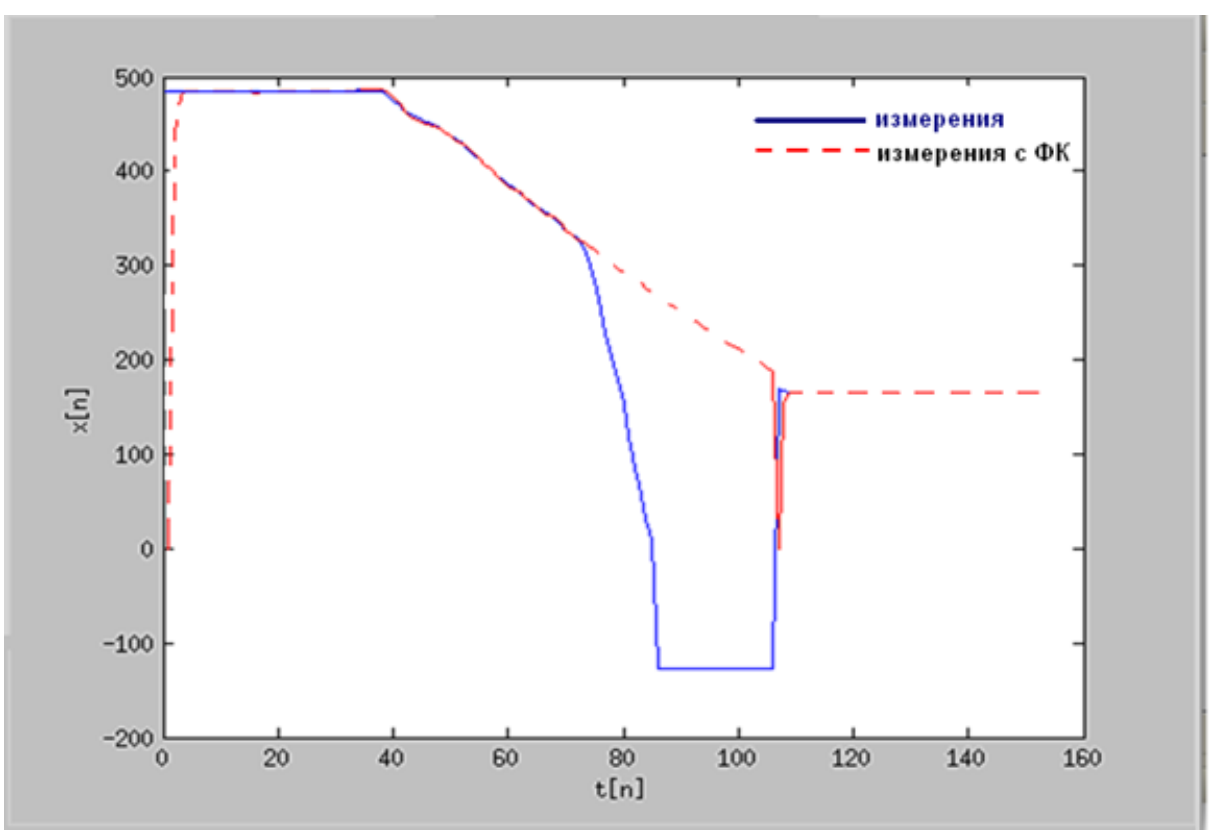

Рис. 5. Данные на выходе ФК при возникновении препятствия и движении камеры 


\section{Повышение надежности распознавания ориентира}

Мы провели ряд исследований для сравнительного анализа надежности распознавания ориентира известными алгоритмами и синтезированным алгоритмом при одинаковых условиях зашумленности. Нами получены следующие результаты (табл. 1):

Таблица 1.

Сравнительный анализ надежности работы алгоритмов

\begin{tabular}{|c|c|c|c|}
\hline $\begin{array}{c}\text { Алгоритм } \\
\text { распознавания }\end{array}$ & $\begin{array}{c}\text { Количество } \\
\text { итераций }\end{array}$ & $\begin{array}{c}\text { Количество } \\
\text { распознанных кадров }\end{array}$ & $\begin{array}{c}\text { Надежность } \\
\text { работы, } \%\end{array}$ \\
\hline
\end{tabular}

Существующие алгоритмы

Синтезированный алгоритм
21

30

Таким образом, надежность синтезированного алгоритма с использованием ФК при возникновении случайных выбросов точек (рис. 7) и изменении уровня освещенности (рис. 9) выше на 30\%. Полученные результаты отображают корректность и надежность распознавания ориентира.

Работа алгоритма (рис. 10) начинается с прогнозирования позиции ключевой точки с использованием ФК.

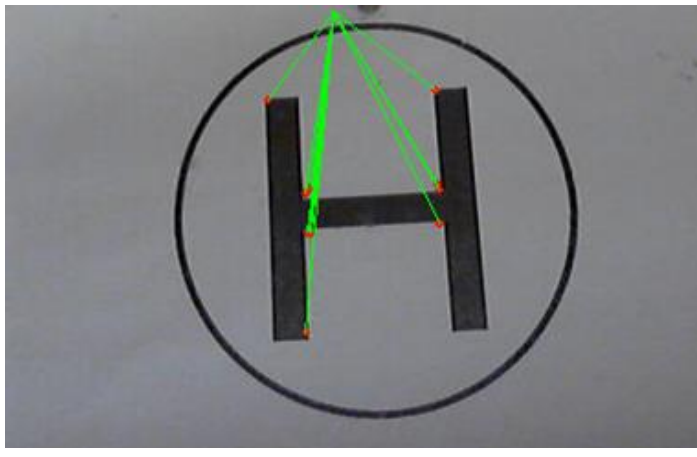

Рис. 6. Результат при неподвижной камере

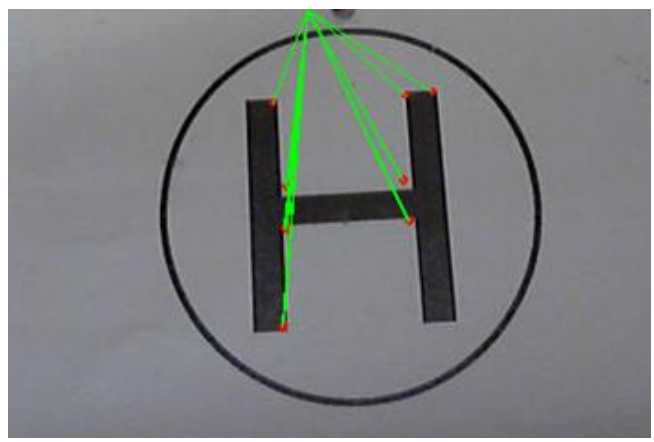

Рис. 8. Результат распознавания при нормальном уровне освещения

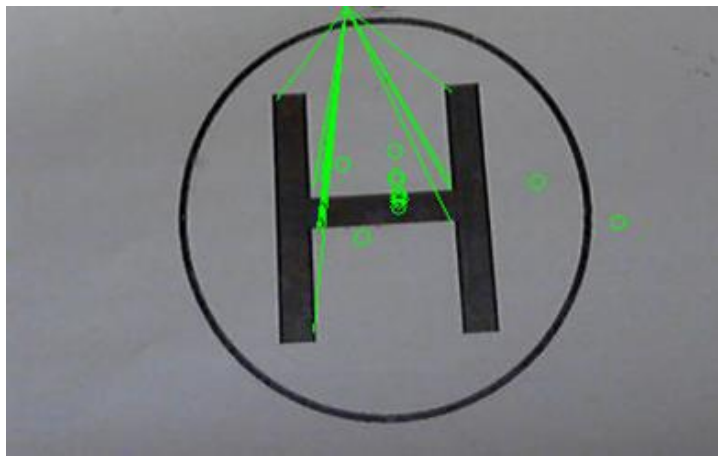

Рис. 7. Результат распознавания при перемещении камеры

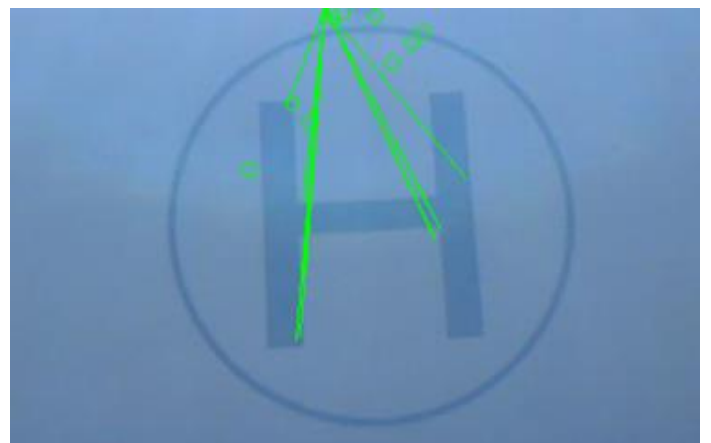

Рис. 9. Результат распознавания при изменении уровня освещения 
перезагрузка сигнала

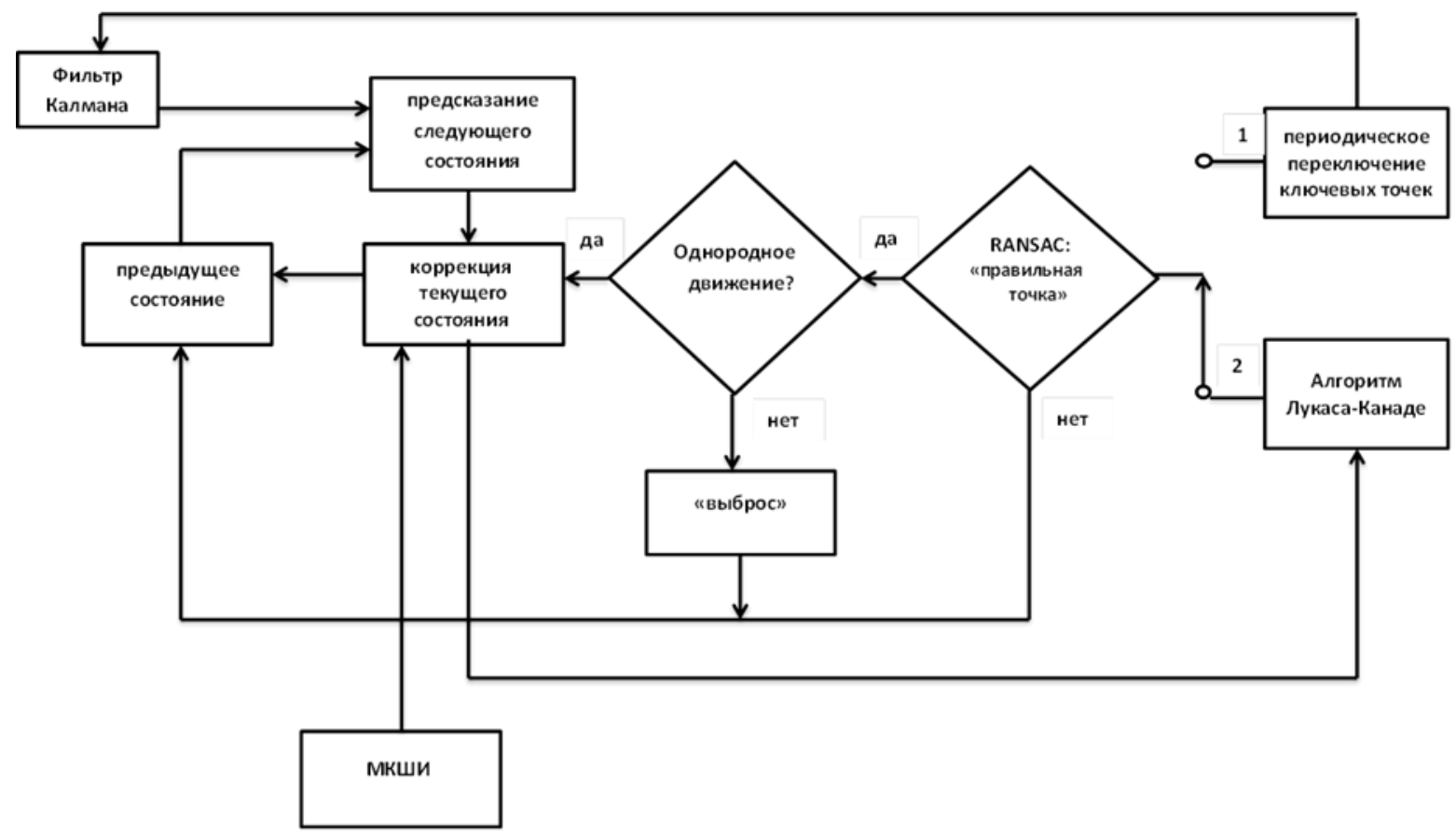

Рис. 10. Блок-схема алгоритма распознавания ориентира

На следующем этапе выполняется коррекция прогнозируемого состояния ключевой точки при проведении измерений. Положение ключевых точек корректируем алгоритмом Лукаса-Канаде. С помощью алгоритма RANSAC проверяем корректность положения ключевых точек, их направление и скорость перемещения. Ключевые точки, которые считаются «выбросами» не используются на этапе коррекции. При отклонении от ориентира и появлении шумов происходит коррекция измерений ковариационной матрицей состояния ключевых точек в блоке КМИ. Новый полученный коэффициент используем для корректировки прогноза состояния ключевой точки. Далее выполняется итерация процесса.

\section{Выводы}

Синтезирован алгоритм распознавания ориентира, который основан на комплексировании известных методов распознавания и применении фильтра Калмана. Методы распознавания скомплексированы таким образом, что при первичной обработке изображения определяется контур ориентира по $h u$-моментам с последующим исключением совпадения контуров методом Лукаса-Канаде и определением особых точек преобразованием Хафа. Осуществляется проверка корректности положения ключевых точек, их направление и скорость перемещения методом RANSAC. Повышение надежности распознавания ориентира применением фильтра Кал- 
мана при наличии шумов, препятствий и изменении освещения повышает надежность распознавания ориентира на $30 \%$.

\section{Список использованной литературы}

1. Прэтт У. Цифровая обработка изображений / Прэтт У. - М.: Мир, 1982. - Кн. 2. -480 c.

2. Ту Дж. Принципы распознавания образов /Дж. Ту, Р. Гонсалес. - М.: Мир, 1978. - 412 с.

3. Гонсалес Р. Цифровая обработка информации / Р. Гонсалес, Р. Вудс М.: Техносфера, 2005. - $1072 \mathrm{c}$.

4. Применение метода Лукаса-Канаде для вычисления оптического потока () И. О. Сакович, Ю. С. Белов КФ МГТУ им. Н. Э. Баумана, Калуга.

5. Сабельников П. Ю. Сравнение контуров объектов с частично искажённой формой / П. Ю. Сабельников // Journal of Qafqaz University. Mathematics and Computer Science. (Baku). - 2012. - № 34 - C. 47-58.

6. Understanding and Applying Kalman Filtering/ Lindsay Kleeman// Department of Electrical and Computer Systems Engineering Monash University, Clayton.

7. Синицын И. Н. Фильтры Калмана и Пугачева/Учебное пособие. - М.: Университетская книга, Логос. 2006. - 640 с.

8. Achtelik M., Weiss S., Siegwart R. Onboard IMU and monocular vision based control for MAVs in unknown in-and outdoor environments // Robotics and automation (ICRA), 2011 IEEE international conference on. - IEEE, 2011. - P. 3056-3063.

9. Яне Б. Цифровая обработка информации / Б. Яне - М.: Техносфера, 2007. $-584 \mathrm{c}$.

10. Методы компьютерной обработки изображений / [под ред. В. А. Сойфера] - 2-е изд., испр. - М.: Физматлит, 2003. - 784 с.

11. Котвицький Р. С. Метод визначення координат рухомого об`єкту з використанням системи технічного зору / Р. С. Котвицький, Г. В. Сарибога, О. В. Збруцький - Інформаційні системи, механіка та керування. - № 16. - 2017. - с. 71-78. 